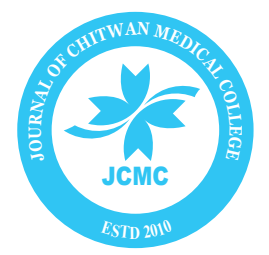

\author{
Journal of Chitwan Medical College 2019;9(30):6-10 \\ Available online at: www.jcmc.cmc.edu.np
}

\title{
SERUM URIC ACID IN PATIENTS WITH ABNORMAL THYROID FUNCTIONS: A CROSS-SECTIONAL STUDY Kushal Bhattarai ${ }^{1,}$, , Nilu Manandhar ${ }^{2}$ \\ ${ }^{1}$ Department of Biochemistry, College of Medical Sciences and Teaching Hospital, Bharatpur, Chitwan, Nepal. ${ }^{2}$ Department of Physiology, College of Medical Sciences and Teaching Hospital, Bharatpur, Chitwan, Nepal.
}



\section{INTRODUCTION}

Uric acid, the end product of purine catabolism, is produced in the body depending on the dietary purine content and the rate of degradation and salvage of purine nucleotides. Typically, the kidneys eliminate around $3 / 5^{\text {th }}$ to $4 / 5^{\text {th }}$ of the body uric acid; the rest is eliminated via different routes, predominantly the gastrointestinal tract. In this regard, hyperuricemia can be the consequence of over-production or under-elimination of uric acid or both. As high as nine-tenth of individuals with hyperuricemia have defective renal handling of uric acid, in the form of decreased glomerular filtration, decreased tubular secretion and enhanced tubular reabsorption. ${ }^{1}$

The thyroid hormones, thyroxine $\left(T_{4}\right)$ and triiodothyronine $\left(T_{3}\right)$ are indispensable to the normal tissue metabolism, in addition to being essential for growth and development. To this end, thyroid dysfunctions manifest in the form of hypo- or hyperthyroidism, characterized by deficient or excessive thyroid hormones and thus, producing clinical evidences of hypo- or hyper-metabolism. ${ }^{2-4}$ As documented by a growing body of evidences, levels of serum uric acid were increased in both hypothyroidism and hyperthyroidism. ${ }^{5-10}$ While hyperuricemia in hypothyroidism is reported to be related to the decreased renal filtration capacity, the same biochemical picture in hyperthyroidism has been attributed to an increased production of uric acid. Persistent hyperuricemia, obviously, is an established risk factor for a number of complications such as gouty arthritis, nephrolithiasis and nephropathies. ${ }^{1}$

On the above grounds, the study was designed to determine the serum uric acid levels and the prevalence rates of hyperuricemia in patients with the history of thyroid dysfunctions (hyper- and hypothyroidism) and to compare these parameters across the gender and age-groups in these patients.

\section{METHODS}

It was a cross-sectional study conducted in the department of Biochemistry, College of Medical Sciences and Teaching Hospital, Bharatpur, Chitwan, Nepal, for a period of four months (April - July, 2019) after obtaining ethical clearance from the Institutional Review Committee of the College of Medical Sciences and Teaching Hospital (COMSTH-IRC) (Ref. No: 2019004).

A non-probability, convenience type of sampling was used for the selection of participants in the study. The optimum sample 
size was calculated using the overall prevalence rate of hyperuricemia in thyroid dysfunction. See et al $^{5}$ reported the prevalence of hyperuricemia in subjects with thyroid dysfunction to be $19.4 \%$. Using this value as the prevalence rate and the margin of error as $0.05(5 \%)$, the minimum sample size was calculated to be 241. However, a small overshoot of the sample size was deemed necessary to account for potential aberrancies in laboratory analysis, non-response of the participants, etc. So the final sample size was 249 .

Adult participants, aged 30 years or older, of both gender (male and female), with the diagnosis of overt hypothyroidism or hyperthyroidism and under medications for at least six months or more, were included, excluding the newly diagnosed ones. Subjects with subclinical hypo- and hyper-thyroidism, and those with self-reported history of intake of drugs that could increase serum uric acid levels were also excluded. After obtaining the written informed consent, data on the demographic variables were obtained by means of personal interview from each study participant who had visited the hospital during the study period. Serum uric acid of the study participants was estimated in the central clinical laboratory in an autoanalyzer. As such, the continuous variables used in the study were (a) age (years) and (b) serum uric acid $(\mathrm{mg} / \mathrm{dL})$; whereas the categorical variables were (a) gender (female/male), (b) age-groups (30-40 years; 40-50 years; $50-60$ years and $>=60$ years), and (c) hyperuricemia (yes/no). Hyperuricemia was defined as serum uric acid concentration of more than $6.8 \mathrm{mg} / \mathrm{dL}$ in males and serum uric acid concentration more than $6.0 \mathrm{mg} / \mathrm{dL}$ in females.

The collected data was first entered into Microsoft Excel 2016. After preliminary refinement, data was again fed into SPSS (Statistical Package for Social Science) version 16.0 for final analysis. Appropriate tables and bar diagrams were utilized for description of the results. Continuous variables were described in the form of mean with standard deviation (SD) whereas, the

Table 1: Status of uric acid in total study participants and according to different subgroups of gender, age and thyroid status

\begin{tabular}{|c|c|c|c|}
\hline & \multicolumn{2}{|c|}{ Hyperuricemia } & \multirow{3}{*}{$\begin{array}{l}\text { Chi-Squared Test } \\
\text { (Significance) }\end{array}$} \\
\hline & No & Yes & \\
\hline & N (\%) & $N(\%)$ & \\
\hline Total Participants ( $n=249)$ & $173(69.5 \%)$ & $76(30.5 \%)$ & - \\
\hline \multicolumn{4}{|l|}{ Thyroid Status } \\
\hline 1. Hyperthyroidism $(n=161)$ & $108(67.1 \%)$ & $53(32.9 \%)$ & \multirow{2}{*}{$p=0.257$} \\
\hline 2. Hypothyroidism $(n=88)$ & $65(73.9 \%)$ & $23(26.1 \%)$ & \\
\hline \multicolumn{4}{|l|}{ Gender } \\
\hline 1. Females $(n=144)$ & 97 (67.4\%) & $47(32.6 \%)$ & \multirow{2}{*}{$p=0.396$} \\
\hline 2. Male $(n=105)$ & $76(72.4 \%)$ & $29(27.6 \%)$ & \\
\hline \multicolumn{4}{|l|}{ Age-Groups } \\
\hline 1. $30-40$ years $(n=57)$ & 41 (71.9\%) & $16(28.1 \%)$ & \multirow{4}{*}{$p=0.954$} \\
\hline 2. $40-50$ years $(n=60)$ & $42(70.0 \%)$ & $18(30.0 \%)$ & \\
\hline 3. $50-60$ years $(n=68)$ & 47 (69.1\%) & $21(30.9 \%)$ & \\
\hline 4. $\geq 60$ years $(n=64)$ & $43(67.2 \%)$ & $21(32.8 \%)$ & \\
\hline
\end{tabular}

categorical variables were depicted as frequency with percentage. For the inferential statistics, non-parametric chi-squared test was used to test the association between various categorical variables. Independent t-test and one-way analysis of variance (ANOVA) were used to test the differences of serum uric acid levels between the various comparison groups. Statistical significance was defined as $p<0.05$ at $95 \%$ confidence intervals.

\section{RESULTS}

In the present study, of the total 255 patients with thyroid dysfunction (hypo- or hyperthyroidism) initially considered for the study, six patients were excluded because of the pre-analytical and analytical aberrancies in laboratory measurement of their serum uric acid. Of the remaining 249 participants, $64.7 \%(n=161)$ had hyperthyroidism and $35.3 \%(n=88)$ had hypothyroidism. Females comprised of $57.8 \%(n=144)$ and males, $42.2 \%(n=105)$ of the total participants. Similarly, of the hyperthyroid subjects, $44.7 \%(n=72)$ were females and $55.3 \%(n=89)$ were males; amongst the hypothyroid subjects, $81.8 \%(n=72)$ were females and $18.2 \%(n=16)$ were males.

Table 1 depicts the prevalence rates of hyperuricemia in the overall participants and in various categories of gender, agegroups and thyroid status. As illustrated, the prevalence of hyperuricemia in thyroid dysfunction was $30.5 \%$. The rate was $32.9 \%$ in hyperthyroid and $26.1 \%$ in hypothyroid subjects. There was no significant association between thyroid status and hyperuricemia amongst thyroid dysfunction patients (chi-squared test; $p=0.257$ ). The prevalence of hyperuricemia was $32.6 \%$ in females and $27.6 \%$ in males. Gender was not significantly associated with hyperuricemia (chi-squared test; $p=0.396)$. Across the different age-groups, the prevalence of the condition was seen to increase with age, with $28.1 \%$ in $30-$ 40 years old subjects (the least) and $32.8 \%$ in subjects older than or equal to 60 years (the highest). Age was not significantly associated with hyperuricemia (chi-squared test; $p=954$ ). 
Table 2: Comparison of the mean serum uric acid concentration $(\mathrm{mg} / \mathrm{dL})$ between different subgroups of gender, age and thyroid status

\begin{tabular}{|c|c|c|c|}
\hline & \multicolumn{2}{|c|}{ Uric Acid (mg/dL) } & \multirow{2}{*}{$\begin{array}{c}\text { Statistical Tests } \\
\text { (Significance) }\end{array}$} \\
\hline & Mean & SD & \\
\hline Total Participants $(n=249)$ & 5.46 & 1.88 & - \\
\hline \multicolumn{4}{|l|}{ Gender } \\
\hline 1. Females $(n=144)$ & 5.43 & 1.91 & \multirow{2}{*}{$\begin{array}{l}\text { Independent t-test } \\
\qquad(p=0.809)\end{array}$} \\
\hline 2. Male $(n=105)$ & 5.49 & 1.84 & \\
\hline \multicolumn{4}{|l|}{ Age-Groups } \\
\hline 1. $30-40$ years $(n=57)$ & 5.21 & 1.88 & \multirow{4}{*}{$\begin{array}{c}\text { Analysis of Variance } \\
\text { (one-way ANOVA) } \\
(p=0.180)\end{array}$} \\
\hline 2. $40-50$ years $(n=60)$ & 5.68 & 1.95 & \\
\hline 3. $50-60$ years $(n=68)$ & 5.19 & 1.69 & \\
\hline 4. $\geq 60$ years $(n=64)$ & 5.76 & 1.96 & \\
\hline \multicolumn{4}{|l|}{ Thyroid Status } \\
\hline 1. Hyperthyroidism $(n=161)$ & 5.60 & 1.71 & \multirow{2}{*}{$\begin{array}{l}\text { Independent } t \text {-test } \\
\qquad(p=0.110)\end{array}$} \\
\hline 2. Hypothyroidism $(n=88)$ & 5.20 & 2.13 & \\
\hline
\end{tabular}

Table 3: Prevalence of hyperuricemia for hyper- and hypothyroidism according to different subgroups of gender and age

\begin{tabular}{|c|c|c|c|c|c|c|}
\hline \multirow{3}{*}{ Variables } & \multicolumn{3}{|c|}{ Hyperthyroidism } & \multicolumn{3}{|c|}{ Hypothyroidism } \\
\hline & \multicolumn{2}{|c|}{ Hyperuricemia } & \multirow{2}{*}{$\begin{array}{l}\text { Significance } \\
\left.\text { ( } \chi^{2} \text { Test }\right)\end{array}$} & \multicolumn{2}{|c|}{ Hyperuricemia } & \multirow{2}{*}{$\begin{array}{c}\text { Significance } \\
\left(\chi^{2} \text { Test }\right) \\
\end{array}$} \\
\hline & No & Yes & & No & Yes & \\
\hline \multicolumn{7}{|l|}{ Gender } \\
\hline 1. Female & $44(61.1 \%)$ & $28(38.9 \%)$ & \multirow{2}{*}{$p=0.147$} & $53(73.6 \%)$ & $19(26.4 \%)$ & \multirow{2}{*}{$p=0.909$} \\
\hline 2. Male & 64 (71.9\%) & $25(28.1 \%)$ & & $12(75.0 \%)$ & $04(25.0 \%)$ & \\
\hline \multicolumn{7}{|c|}{ Age-Groups (years) } \\
\hline 1. $30-40$ & $24(68.6 \%)$ & 11 (31.4\%) & \multirow{4}{*}{$p=0.995$} & $17(77.3 \%)$ & $05(22.7 \%)$ & \multirow{4}{*}{$p=0.921$} \\
\hline 2. $40-50$ & $23(67.6 \%)$ & 11 (32.4\%) & & $19(73.1 \%)$ & 07 (26.9\%) & \\
\hline 3. $50-60$ & $31(66.0 \%)$ & $16(34.0 \%)$ & & $16(76.2 \%)$ & $05(23.8 \%)$ & \\
\hline 4. $\geq 60$ & $30(66.7 \%)$ & $15(33.3 \%)$ & & $13(68.4 \%)$ & $06(31.6 \%)$ & \\
\hline
\end{tabular}

Table 2 depicts the comparison of the mean serum uric acid concentration $(\mathrm{mg} / \mathrm{dL})$ between different categories of gender, agegroups and thyroid status. As shown in the table, the mean serum uric acid levels in overall patients with thyroid dysfunction was $5.46 \pm 1.88 \mathrm{mg} / \mathrm{dL}$. It was slightly higher in male subjects than in the female subjects. However, the difference was not statistically significant (Independent t-test; $p=0.809$ ). Similarly, the mean serum uric acid levels did not vary significantly across the various age-groups (one-way ANOVA; $p=0.180$ ). Subjects with hyperthyroidism had higher mean serum uric acid as compared to the hypothyroid subjects. However, the difference was not statistically significant (Independent t-test; $\mathrm{p}=0.110$ ).

Table 3 and Figure 1 show the prevalence rates of hyperuricemia across the different thyroid status for subgroups of gender (1a) and age (1b). As illustrated in Table 3 and Figure 1a, the prevalence of hyperuricemia was greater in females than males in both groups of thyroid dysfunction (38.9\% vs $28.1 \%$ in hyperthyroidism and $26.4 \%$ vs $25.0 \%$ in hypothyroidism). However, there was no significant association between gender and hyperuricemia in any of these groups $(p>0.05)$. 
Among the hyperthyroid subjects, prevalence of hyperuricemia was the least in 30-40 years old, and the highest in 50-60 years old. Similarly, among the hypothyroid subjects, the rate was lowest in $30-40$ years old, and the highest in $>=60$ years old. There was no significant association between hyperuricemia and age in either of the two groups ( $>>0.05)$ (Table 3 and Figure 1b).

\section{DISCUSSION}

Serum uric acid is a function of rate of purine catabolism and renal tubular secretion and reabsorption. Evidently, hyperuricemia is secondary to an alteration in any of these; increased purine catabolism or decreased secretion or increased tubular reabsorption. Thyroid dysfunctions manifest in the form of either increased or decreased levels of thyroid hormones. While hypermetabolism is key to the former, the opposite effects are characteristic for the later. As a matter of fact, a growing body of evidences reporting the possible relationships between thyroid dysfunction and hyperuricemia prompted the present study.

The overall prevalence of hyperuricemia in the present study was $30.5 \%$. It was $32.6 \%$ in female and $27.6 \%$ in male participants, with no statistically significant association of hyperuricemia with gender $(p>0.05)$. However, the mean serum uric acid concentration was marginally greater in the males than in the females, albeit the difference was not statistically significant $(p>0.05)$. Serum concentration of uric acid has been reported to be low in females, especially during the pre-menopausal age. However, post-menopausal females have been found to show increased concentrations as compared to the males of the same age-groups. ${ }^{1}$ In this study, the prevalence of hyperuricemia was found to increase with age, with the lowest rate of $28.1 \%$ in the youngest and the highest rate of $32.8 \%$ in the eldest subjects. However, age was not significantly associated with hyperuricemia ( $p>0.05$ ). Likewise, the mean serum uric acid levels, too, did not vary significantly across the various age-groups ( $p>0.05)$.

In the present study, the prevalence rates of hyperuricemia in hyperthyroid and hypothyroid subjects were respectively, $32.9 \%$ and $26.1 \%$ (overall prevalence: $30.5 \%$ ), with no significant association between thyroid status and hyperuricemia ( $p>0.05$ ). Mean serum uric acid was higher in the hyperthyroid subjects than in the hypothyroid ones. However, the difference was not significant $(p>0.05)$. Different investigators have found increased prevalence rates of hyperuricemia in subjects with thyroid dysfunctions. In their study, See et al $^{5}$ reported the prevalence of hyperuricemia in subjects with thyroid dysfunction to be $19.4 \%$. They further affirmed higher prevalence of hyperuricemia and gout in participants with hypo- and hyperthyroidisms than the euthyroid ones. ${ }^{5}$ Other investigators such as Giordano et al $^{6}$ have also reported a significantly higher occurrence of hyperuricemia in hyperthyroid and hyperuricemia with gout in hypothyroid patients as compared to the general population. Jia et $\mathrm{al}^{7}$ have reported significantly higher $(\mathrm{p}<0.05)$ serum levels of uric acid in the overt hypothyroid than in the euthyroid or subclinically hypothyroid participants. Similarly, Sato et $\mathrm{al}^{8}$ evaluated the relationship between serum thyroxine and uric acid in the hyperthyroid patients and found significantly elevated serum uric acid in such patients. Ye et al $^{9}$ studied the association between thyroid status and serum concentrations of uric acid in participants with subclinical hyperthyroidism. In such subjects, after controlling for possible covariates, serum thyroxine and uric acid demonstrated statistically significant positive and linear association $(p<0.001)$.

Verhelst et al, ${ }^{10}$ in their study, concluded significantly increased GFR (glomerular filtration rate) in hyperthyroid and significantly decreased GFR in hypothyroid subjects $(p<0.05)$. This points towards the effect of thyroid dysfunction in kidney functions. Increased concentration of thyroid hormones (in hyperthyroidism) may lower the serum creatinine by increasing its renal clearance. Similarly, in hypothyroidism, clearance decreases, probably leading to the retention of this nitrogenous compound in the circulation. To this end, den Hollander et al ${ }^{11}$ assessed the relationship between kidney function and thyroid status before and after treatment for hypo- and hyperthyroidism, and found a strong correlation between change in thyroid status and kidney function $(p<0.001)$. Jia et $\mathrm{al}^{7}$ also found a significantly lower $(p<0.05)$ GFR in the hypothyroid than in the euthyroid subjects. With this background, it is quite plausible to attribute hyperuricemia in hypothyroid subjects to the decreased renal clearance of uric acid. Giordano et $\mathrm{al}^{6}{ }^{6}$ in their study, have attributed hyperuricemia to increased formation of uric acid in hyperthyroid and decreased renal clearance in hypothyroid subjects.

In some of the studies, serum uric acid levels failed to show any significant elevation, especially in the pediatric patients with subclinical hypothyroidism. In one such study, Sayari et al $^{12}$ found that children with subclinical hypothyroidism did not have significantly different serum uric acid levels ( $p>0.05)$, although serum creatinine levels were significantly elevated, signifying a decline in the clearance of this substance.

In the present study, the prevalence of hyperuricemia was greater in females than males in both groups of thyroid dysfunction (38.9\% vs $28.1 \%$ in hyperthyroidism and $26.4 \%$ vs $25.0 \%$ in hypothyroidism). However, there was no significant association between gender and hyperuricemia in any of these groups $(p>0.05)$. In a cross-sectional study, Zhang et $\mathrm{al}^{13}$ reported that males with subclinical hypothyroidism had a significantly higher prevalence of hyperuricemia than the females $(p<0.05)$. Moreover, these male participants with the condition showed significantly increased odds of having hyperuricemia as compared to those without the condition. However, in the females, no such association was seen. To add, both gender groups with subclinical hypothyroidism had significantly decreased estimated GFR values. Explicably, this parameter was significantly greater in subclinically hyperthyroid males and females alike.

Among the hyperthyroid subjects, prevalence of hyperuricemia was the least in 30-40 years old, and the highest in 50-60 years old. Similarly, among the hypothyroid subjects, the rate was lowest in $30-40$ years old, and the highest in $>=60$ years 
old. Although, there was no significant association between hyperuricemia and age in either of the two groups ( $p>0.05)$, the trend throws some light onto the age-related changes in uric acid homeostasis.

In this study, thyroid status was based on reported history only and may suffer from self-report bias. In this matter, relationship of thyrotropin and/or thyroid hormones with serum uric acid could have been assessed to yield more valuable information. Moreover, only the serum uric acid was evaluated as a biochemical parameter in the present study. An expounded panel consisting of other parameters, such as serum creatinine for calculation of estimated GFR, and fractional excretion of uric acid do provide some valuable and concrete information about the cause of hyperuricemia in the subjects with thyroid dysfunction. Ideally, estimation of the amount of uric acid excreted in urine in a 24-hour sample can be used to determine whether hyperuricemia is caused by overproduction or by decreased excretion. ${ }^{1}$ Further, subjects with subclinical thyroid dysfunctions were excluded, due to the paucity of samples with such conditions. A study with larger samples and inclusion of these subjects adds more evidence to the study. To add more, possible covariates that may affect serum uric acid levels were missing in the study. Information on personal habits such mellitus, hypertension could have been included to strongly assert the association between the primary variables of interest as alcohol intake and certain medical conditions like diabetes

in the present study. Lastly, it was a hospital-based study with limited generalizability of the results obtained. On top of that, a cross-sectional study such as this always suffers from an inherent limitation of causal relationship.

\section{CONCLUSION}

Although statistically non-significant, the prevalence rates of hyperuricemia and mean serum uric acid levels were higher in patients with hyperthyroidism than the hypothyroidism. Moreover, females with both hypo- and hyperthyroidism had higher rates of hyperuricemia than the males. Lastly, the youngest subjects with both thyroid dysfunctions had the lowest rates of hyperuricemia and the rate showed a fairly increasing trends with age. These findings point towards some insights into the mechanisms of development of hyperuricemia and thus, clearly warrants some interventional approaches. More studies with longitudinal design and with additional variables can be planned for understanding the causal relationship between thyroid status and serum uric acid.

\section{CONFLICT OF INTEREST}

None

\section{FINANCIAL DISCLOSURE}

None

tween Serum Uric Acid and Creatinine in Patients with Hypothyroidism Journal of Sichuan University Medical science. 2015;46(5):747-9. [PMID]

1. Burns CM, Wortmann RL. Disorders of Purine and Pyrimidine Metabolism. In: Kasper DL, Hauser SL, Jameson JL, Fauci AS, Longo DL, Loscalzo J, editor. Harrison's Principles of Internal Medicine. 19th ed. New York: McGraw Hill Education; 2012. p. 431e1-e6.

2. Jameson JL, Mandel SJ, Weetman AP. Disorders of the Thyroid Gland. In: Kasper DL, Hauser SL, Jameson JL, Fauci AS, Longo DL, Loscalzo J, editor. Harrison's Principles of Internal Medicine. 19th ed. New York: McGraw Hill Education; 2015. p. 2283-308.

3. Winter WE, Schatz D, Bertholf RL. The Thyroid: Pathophysiology and Thyroid Function Testing. In: Burtis CA, Ashwood ER, Bruns DE, editor. Tietz Textbook of Clinical Chemistry and Molecular Diagnostics. 5th ed. St. Louis, Missouri: Elsevier Saunders; 2012. p. 1905-44.

4. The Thyroid Gland. In: Barret KE, Barman SM, Boitano S, Brooks HL, editor. Ganog's Review of Medical Physiology. 25th ed. New York: McGraw Hill Education; 2016. p. 337-50.

5. See LC, Kuo CF, Yu KH, Luo SF, Chou IJ, Ko YS, et al. Hyperthyroid and hypothyroid status was strongly associated with gout and weakly associated with hyperuricaemia. PloS one. 2014;9(12):e114579. [DOI]

6. Giordano N, Santacroce C, Mattii G, Geraci S, Amendola A, Gennari C. Hyperuricemia and gout in thyroid endocrine disorders. Clinical and experimental rheumatology. 2001;19(6):661-5. [PMID]

7. Jia D, Liang LB, Tang GH, He H, Zhang M, Li ZP, et al. The Association Be-
8. Sato A, Shirota T, Shinoda T, Komiya I, Aizawa T, Takemura Y, et al. Hyperuricemia in patients with hyperthyroidism due to Graves' disease. Metabolism: clinical and experimental. 1995;44(2):207-11. [DOI]

9. Ye $Y$, Gai X, Xie H, Jiao L, Zhang S. Association between serum free thyroxine (FT4) and uric acid levels in populations without overt thyroid dysfunction. Annals of clinical and laboratory science. 2015;45(1):49-53. [PMID]

10. Verhelst J, Berwaerts J, Marescau B, Abs R, Neels H, Mahler C, et al. Serum creatine, creatinine, and other guanidino compounds in patients with thyroid dysfunction. Metabolism: clinical and experimental. 1997;46(9):1063-7. [DOI]

11. den Hollander JG, Wulkan RW, Mantel MJ, Berghout A. Correlation between severity of thyroid dysfunction and renal function. Clinical endocrinology. 2005;62(4):423-7. [DOI]

12. Sayari S, Molaei Z, Torabi Z. The relationship between subclinical hypothyroidism and serum levels of uric acid and creatinine in children aged 2-14 years. Annals of pediatric endocrinology \& metabolism. 2018;23(1):38 42. [DOI]

13. Zhang J, Meng Z, Zhang Q, Liu L, Song K, Tan J, et al. Gender impact on the correlations between subclinical thyroid dysfunction and hyperuricemia in Chinese. Clinical rheumatology. 2016;35(1):143-9. [DOI] 